\title{
The Impact of Big Data on Business Management Decisions
}

\author{
Liu Yunpeng \\ Xijing University, Shaanxi Xi'an 710123, China
}

Keywords: big data; management decision; impact;

Abstract: Based on the background of big data, the form of enterprise management decision-making has undergone earth-shaking transformation. Big data has a lot of data resources, providing decision-makers with a reference platform for them to better find data and improve management decision-making skills. At the same time, it puts forward higher requirements for enterprise management decision-making. Enterprise management decision-makers need to innovate their own ideas and ideas, follow up the reforms, and meet the development needs of big data. This paper briefly summarizes the big data information, clarifies the problems of enterprise management decision in the big data environment, and proposes solutions. Finally, it analyzes the impact of big data on enterprise management decisions.

\section{Introduction}

For enterprises, correct management decisions are the key channel to improve overall strength. The lifeblood of an enterprise is management decision-making, and the level of management decisions determines the economic profit of the enterprise. Rich data resources make it easier for enterprises to find data faster and innovate the decision-making atmosphere of enterprise management. With the expansion of data scale, the data relationship is very close. At this time, enterprises must adapt to the trend of social development, improve the level of corporate management decision-making, and stabilize the position of enterprises in the market competition.

\section{Overview of big data}

\subsection{Meaning}

Big data has certain functionality. It is a huge collection of data. It is understood from the "Huge" that big data sets are very large in both quantity and scale, breaking people's traditional cognition. New software tools and hardware devices must be used for big data collection, storage, management, and research. Otherwise, general equipment tools cannot meet the requirements of big data research functions. The meaning of big data: large scale, fast spread, and many types. Big Data Technology: The technology used in big data, big data technology that can be collected, managed and applied in a short period of time. Since the launch of big data, it has attracted people's attention and is widely used in various collections, fully demonstrating the value of big data technology. 


\subsection{The features of big data}

\subsubsection{Large amount of data}

The large amount of data is one of the most notable features of big data. Compared with general data, the amount of data of big data can be summarized by "Huge". Current regular data files are still in GB units, and big data has risen to the TB level. Especially in commercial areas, many retail companies have risen to the terabyte level in production.

\subsubsection{Spread fast}

Big data has a lot of information data, so the speed propagation requirements in the data processing are also relatively high. In recent years, information technology has been continuously developed and innovated. Mobile communication technology and Internet technology have been widely used in various applications around the world, speeding up the frequency of data generation and transmission, and promoting the development of big data technology. The value of big data technology is fully reflected in the speed of data generation and propagation.

\subsubsection{Many types}

With the advent of the information technology era, the variety of data is increasing. Overall, data can be divided into two types, structured data and unstructured data. Structured data is the data formed under the premise of basic behavior. The production process of this kind of data is the same, so the data structure is very similar. In the process of analyzing and processing data, the specified pattern should be processed, and then the data information can be quickly obtained. For unstructured data, its production form is diversified, and it can create many unstructured data regardless of network clicks, document transmission, and radio frequency identification.

\section{Problems in enterprise management decision-making under the big data environment}

\subsection{Backward decision-making method}

With the advent of the information age, companies have made decision-making plans based on decision-making data. Today's big data analysis and traditional logical reasoning are very different. Therefore, large-scale search, comparison and classification processing of batch data is required, and data relevance is emphasized. The new decision management system can quickly find key data in batch data and determine the relationship between data. To clearly describe the data relevance, it is necessary to use the degree of trust and support to complete the description. In the process of exploring the value of data, if you can use some way to improve the company's income, you can support enterprise management decisions. The relevance of data holding requires managers to combine business situations and innovative decision-making methods.

\subsection{The decision-making body is characterized by diversity}

In the modern society, enterprises must continuously enhance decision-making technology and professional knowledge, and expand the scale of data. Not only do they need enterprise management personnel, but also scholars, professional and technical personnel. There are many types of corporate decision-making entities, which are diversified. The diversification of decision-making bodies can greatly improve the decision-making rate of enterprises. Based on this, enterprises should quickly create a decision-making management system, collect data efficiently, organize data, and provide 
quality services for enterprise management decisions.

\subsection{Decision process lag}

The decision-making process is very cumbersome in traditional enterprises, and enterprises need to spend a lot of time collecting, organizing information and selecting evaluation plans. Such factors make the degree of decision-making of enterprises lag behind and lose opportunities. Based on the information age, the company's top priority is to go to the redundant part of the decision-making process, “preemptive strike”, make quick decisions, and stabilize the market position. In other words, the later corporate competition is based on big data competition. Using big data technology, companies can better explore the value of information, find favorable parts of corporate decision-making, and finally make scientific judgments.

\subsection{Decision information identification difficulties}

With the advent of the information age, Internet data is on the rise, and the annual data volume has reached the current ZB level from the beginning of the terabytes. The data contains infinite value. Traditional data management and processing techniques are difficult to explore the value of data, making it impossible for business managers to accurately determine the value of data. Based on this, we actively introduce new technologies and create a functional scale large enterprise management decision system. In this way, companies can obtain more information and data, formulate decision-making plans, and achieve sustainable development goals.

\section{Enterprise management decision-making method under data environment}

\subsection{Create a systematic big data integration system}

Due to the influence of big data, the decision-making environment of enterprises is very cumbersome. In order to solve such phenomena and reduce the difficulty of decision-making, enterprises need to establish a systematic big data integration system. Combined with cloud computing technology and big data technology, all kinds of information can be quickly judged, and enterprises can more accurately grasp their environmental changes. The big data integration system can meet enterprise data processing requirements, and use scalability and practicality to complete decision tasks. Based on the big data integration processing system, enterprises can collect market operation information, consumer information and product sales information in a targeted manner. This information can reflect its own value with the help of the system, understand consumer requirements, and rationally formulate business plans; The product structure is consistent with the market structure to meet consumer demand.

\subsection{Building a corporate management decision culture}

With different decision-making cultures, companies often choose different decision-making models to achieve different decision-making effects. Decision-making culture influences the value concept of decision makers, and the decision-making process and decision-making effects change. Under normal circumstances, corporate decision-making is attached to senior management, subjective and heavy, resulting in a corporate decision-making culture. Because of the limited capacity of senior management, the information held by them is not very comprehensive, thus increasing the risk of decision-making process. With the help of big data technology, companies hold a lot of decision-making information, improve information analysis capabilities and forecast 
accuracy, so as to make correct judgments and promote enterprises to establish a data-driven decision-making culture.

\subsection{Reasonable choice of decision-making thinking mode}

The decision-making body's thinking mode determines the decision-making result, and the different decision-making subjects present different basis, and the final decision-making result will also be affected. Based on the big data technology environment, enterprises can expand the scope of information search, introduce other mathematical modeling methods and computer technology and in-depth study of data, and implement predictions on the probability of occurrence of events, and fundamentally improve the rationality of decision-making results. Based on this, the decision-making body of the enterprise needs to select a rigorous decision-making thinking mode, and change the traditional subjective and empirical decision-making thinking mode into a decision-making thinking mode in which data and fact coexist. It is the thinking characteristic of big data enterprise management decision-making. According to the data, the implementation of corporate decision-making based on the thinking mode, the operational and financial situation is true. In addition, corporate managers need to strengthen their data value awareness, dig deeper into data values, and effectively support decision-making.

\subsection{Decentralized enterprise management decision-making power}

Once the main body of enterprise management decision has changed, it is necessary to adjust the allocation process of enterprise management decision-making power. Based on the view of big data, there are many uncertain factors in enterprise management decision-making, which leads to a series of decentralized features in decision-making power distribution. In other words, enterprise management decision-making power is biased toward general managers and grassroots workers. The decision-making information that enterprises can use continues to rise, which has pushed the decision-making quality of enterprises to the direction of flattening. In addition to corporate executives, general managers and grassroots workers can use this information to enhance the decentralization of corporate management decision-making power. The existing pyramid-type decision structure of the enterprise is transformed into a network structure. Based on this, the distribution of decision-making power should be similar to the organizational structure. The distribution of decentralized decision-making power gradually replaces the traditional centralized decision-making power distribution, giving the general managers and grass-roots employees greater decision-making rights.

\section{The impact of big data on business management decisions}

Big data technology is developing rapidly, and it is widely used in various industries. Scientific use of big data technology can deeply explore user behavior and product sales, so as to predict market development trends in a short period of time, so that enterprises can support data in management decisions. In general, the impact of big data on business management decisions is reflected in the following points.

\subsection{Management decision environment}

Since the introduction of big data technology, many companies have paid more and more attention to big data technology, and they have studied enterprise data and industry technology through big data technology, thus helping enterprise management to obtain real and safe industry information 
data and let management to the industry environment. Have a general understanding. Nowadays, big data technology is widely used in many large enterprises in China, which has promoted the development process of the enterprise to some extent. For enterprises, some small and medium-sized enterprises pay less attention to big data applications. The reason for such phenomena is that corporate costs are constrained, or the management of the company ignores the development of the industry. If the enterprise can scientifically use big data technology in the development stage and conform to the development trend of the times, it will certainly have a better future in the later development process.

\subsection{Management Decision Data}

Any enterprise management decision-making work is very complicated, and managers must fully understand the development of the enterprise and the industry in the decision-making stage. In the past, in the process of enterprise management decision-making, management often used some documents and operational data for analysis in the process of understanding the enterprise. Such files and data represent enterprise management decision data. The amount of information in such data is very large, and the information is deeply hidden. If it is lack of professionalism and forward-looking, it will increase the difficulty of data screening. With the wide application of big data technology, managers can analyze industry information data in a short period of time, and provide guarantee for the precise security of decision data, which helps managers to better complete enterprise management decision-making tasks.

\subsection{Management Decision Participants}

In past corporate decision-making meetings, the people who usually attended the meeting were leaders, and they affected the development direction of the company to a certain extent. However, these leaders are not very clear about the internal data of the company, so that if the company submits the report data directly at the meeting, it cannot guarantee that all participating leaders can intuitively understand the development of the enterprise. In order to ensure that everyone can clearly understand the industry situation and the direction of enterprise development, professional data analysts should be hired to analyze the company's recent data reports and industry trends. The purpose of this is to enable all participants to understand the industry. A small number of enterprises in the decision-making process due to the implementation of data collection, processing and analysis work, resulting in decision-making personnel can only accomplish this task in decision-making, which intensifies the late development risk of the enterprise. With the advent of big data technology, enterprises are more simple in data collection, storage and analysis. Under the support of scientific data, enterprise managers can make decisions more efficiently and scientifically.

\subsection{Enterprise Risk Management}

Nowadays, China's economic construction is advancing by leaps and bounds, which has intensified the competition between society and enterprises. The competition between enterprises and enterprises in the same industry is equally cruel. If the decision of the enterprise manager is wrong, it will greatly reduce the economic benefits of the enterprise, and more seriously will make the enterprise face bankruptcy. There are many risk factors in the development stage of the enterprise, such as economic risk, resource risk, policy risk and competitive risk. For enterprise risk management, management needs attention. In the past, enterprise risk management was based on the management's long-term experience in enterprise management, and lacked a safe risk prediction method and risk control method. With the rapid development of big data technology, enterprise management can use 
data analysis to understand the development of the company and make judgments. After in-depth discussion of the company's long-term profit data and sales data, comprehensive comparison of corporate development trends, integration of industry market conditions for analysis, and then the future development of the company. After comparing enterprise data information and financial data, real-time monitoring of internal financial risks and operational risks ensures that the company operates in a safe and reliable environment. The use of big data technology involves all aspects of the enterprise, which helps the rapid development of the enterprise. The management can use the big data technology to further strengthen the management and control of the enterprise risk and ensure the long-term stable development of the enterprise.

\subsection{Decision Management Technology}

Enterprise management decision-making technology is affected by the big data environment. Compared with the basic enterprise data processing and analysis, the previous enterprise data analysis and processing work is completed by the corresponding department heads to find the problems behind the data. Management can combine data to implement decisions. The use of big data technology can save this layer of links. In addition, the role of big data technology is not only reflected in the data analysis at the basic level, but also helps the management to better apply the data. Through the comprehensive comparison of the data, combined with the development of the industry, it can effectively measure the customer. The preference, the distribution of potential customers and the development trend of the industry ensure that the decision-making of the enterprise is more flexible and changeable, which has greatly helped the development of the enterprise.

\section{Conclusion}

In summary, based on the background of big data, the form of enterprise management decision-making has undergone earth-shaking transformation. Big data has a lot of data resources, providing decision makers with a reference platform for them to better find data. The introduction of big data is not a simple implementation, but with the advancement of information technology, it can be better integrated into people's daily work and life, and then generate large amounts of data information, which involves all aspects of people. In other words, a person will form a lot of data information from the beginning of his birth. Scientific use of this data information can help companies better select customers and enable the management to make targeted decisions and ensure the company's Long-term stable development.

\section{References}

[1] Chen Xin. Analysis of the Impact of Big Data on Enterprise Management Decisions [J]. Enterprise Reform and Management, 2016, 16(25): 8-9.

[2] Feng Zhiyan, Guo Xunhua, Zeng Dajun. Research and Discussion on the Impact of Big Data on Business Management Decision-making [J]. Science and Science and Technology Management, 2016, (3): 146-149.

[3] Li Gang, Yu Haixu. Challenges and Countermeasures of Enterprise Management in the Age of Big Data [J]. Management and Management, 2017, (39): 56-58.

[4] Liu Shuping. Research on Enterprise Management Model Innovation in the Age of Big Data [J]. Tax, 2017(16): 74-75.

[5] Fan Ning, Zou Rong, Zhang Enying. Analysis of the Application of Big Data in Enterprise Management [J]. Modern Business, 2017 (1): 169-170.

[6] Chen Yunlong. Analysis of Knowledge Management in Big Data Decision Making [J]. Leadership Science Forum, 2017(7): 81-82. 